\title{
Construction of Rational Curves with Rational Rotation-Minimizing Frames via Möbius Transformations
}

\author{
Michael Bartoñ ${ }^{1}$, Bert Jüttler ${ }^{2}$, and Wenping Wang ${ }^{3}$ \\ 1 Technion - Israel Institute of Technology, Haifa, Israel \\ 2 Institute of Applied Geometry, Johannes Kepler University, Linz, Austria \\ 3 Dept. of Computer Science, University of Hongkong, China
}

\begin{abstract}
We show that Möbius transformations preserve the rotationminimizing frames which are associated with space curves. In addition, these transformations are known to preserve the class of rational Pythagorean-hodograph curves and also rational frames. Based on these observations we derive an algorithm for $G^{1}$ Hermite interpolation by rational Pythagorean-hodograph curves with rational rotation-minimizing frames.
\end{abstract}

Key words: rational rotation-minimizing frame, Möbius transformations, Pythagorean-hodograph curve

\section{Introduction}

The rotation-minimizing frames which are associated with a space curve are useful for generating sweep surfaces by moving a profile curve along a trajectory. The first publication discussing this frame was probably [4]. Since then, the computation of this frame and of the resulting sweep surfaces has been discussed in a substantial number of publications, see [23] and the references cited therein. In particular, the construction of rational parameterizations of these sweep surfaces is of interest, due to the importance of NURBS representations for Computer-Aided Design.

The class of spatial Pythagorean-hodograph $(\mathrm{PH})$ curves, which was introduced in [10], seems to be particularly useful for sweep surface modeling. See [8] for an introduction to this topic with many related references. These curves are equipped with rational frames [14], which are called the Euler-Rodrigues frames $[5,6]$. In addition, certain PH curves support the exact computation of rotation-minimizing frames via integration of rational functions [7].

PH space curves with rational rotation-minimizing frames have also been analyzed in the literature. While such frames do not exist on PH cubics [12], an example of a spatial $\mathrm{PH}$ curve of degree 7 with a rational rotation-minimizing frame has been reported in [5]. In that paper, the authors analyzed the conditions under which the Euler-Rodrigues frame of a PH curve is also a rotation-minimizing frame, and they showed that this requires at least polynomial degree 7 for space 
curves. In a recent manuscript, [9], the authors use the Hopf map representation for $\mathrm{PH}$ space curves to demonstrate the existence of rational rotation-minimizing frames on spatial PH quintics.

The surfaces generated by rotation-minimizing frames have been called profile surfaces in $[18,19]$. Based on the kinematical interpretation of rotationminimizing frames as plane-rolling motions, a construction for rational profile surfaces has been presented in [19]. This construction gives rational space curves with rational rotation-minimizing frames, but the degree of the frames - and hence of the resulting profile surfaces - is relatively high for non-planar curves.

Möbius transformations map polynomial or rational $\mathrm{PH}$ curves into rational $\mathrm{PH}$ curves [20], where a rational $\mathrm{PH}$ curve is defined as a curve with rational parametric speed. In addition to this result, in the present paper we show that Möbius transformations preserve rotation-minimizing frames, and in particular rational ones. This observation greatly enhances the flexibility of existing constructions for curves with rational rotation-minimizing frames [5,9], since the group of Möbius transformations can be used to generate new ones.

We demonstrate this observation by formulating an algorithm for $G^{1}$ Hermite interpolation by rational $\mathrm{PH}$ curves with rational rotation-minimizing frames. It is based on Möbius transformations which are applied to planar PH cubics. In the case of the latter curves, the Frenet frames are trivially rotation-minimizing and also rational.

\section{Rotation-minimizing frames \& Möbius transformations}

We recall the notion of the rotation-minimizing frame which can be associated with any space curve and summarize the properties of Möbius transformations. We then analyze the behaviour of rotation-minimizing frames, of Pythagoreanhodograph curves, and of curves with rational rotation-minimizing frames under Möbius transformations.

\subsection{Rotation-minimizing frames}

We consider a $C^{\infty}$ parametric curve segment $\mathbf{x}: u \mapsto \mathbf{x}(u)$ with the parameter domain $I=[0,1]$. We assume that $\mathbf{x}$ is regular for all $u \in I$, i.e., $\mathbf{x}^{\prime}(u) \neq \mathbf{0}$, where the prime' indicates the differentiation with respect to $u$.

We consider a vector field $\mathbf{r}: u \mapsto \mathbf{r}(u)$ along the curve $\mathbf{x}$. Such a vector field is said to be a rotation-minimizing with respect to $\mathbf{x}$ if there exists a function $\lambda$ such that $\mathbf{r}$ satisfies the equations

$$
\left.\begin{array}{rl}
\mathbf{r}^{\prime}(u)-\lambda(u) \mathbf{x}^{\prime}(u) & =0 \\
\mathbf{x}^{\prime}(u) \cdot \mathbf{r}(u) & =0
\end{array}\right\}
$$

for all $u \in I$. The first equation implies $\lambda=\left(\mathbf{r}^{\prime} \cdot \mathbf{x}^{\prime}\right) /\left(\mathbf{x}^{\prime} \cdot \mathbf{x}^{\prime}\right)$. The second equation expresses the fact that the vector $\mathbf{r}(u)$ is contained in the normal plane of the curve at $\mathbf{x}(u)$. Due to

$$
(\mathbf{r}(u) \cdot \mathbf{r}(u))^{\prime}=2 \mathbf{r}^{\prime}(u) \cdot \mathbf{r}(u)=2 \lambda(u) \mathbf{x}^{\prime}(u) \cdot \mathbf{r}(u)=0,
$$


the length of the rotation-minimizing vectors $\mathbf{r}(u)$ is constant.

A rotation-minimizing frame (RMF) of the given curve $\mathbf{x}$ is defined as a triplet of vectors

$$
u \mapsto(\mathbf{t}(u), \mathbf{r}(u), \mathbf{s}(u))
$$

with a unit vector $\mathbf{r}(u)$, where $\mathbf{t}=\mathbf{x}^{\prime} /\left|\mathbf{x}^{\prime}\right|$ is the unit tangent vector of the given curve and $\mathbf{s}(u)=\mathbf{t}(u) \times \mathbf{r}(u)$. Any vector field $\varrho \mathbf{r}(u)+\sigma \mathbf{s}(u)$ with constant coefficients $\varrho, \sigma$ is obviously again rotation-minimizing.

Let

$$
F_{\mathbf{x}}=\left\{\mathbf{w}, \mathbf{w} \cdot \mathbf{x}^{\prime}(0)=0,\|\mathbf{w}\|=1\right\}
$$

be the unit circle in the normal plane of the curve at $\mathbf{x}(0)$, which is also the set of feasible initial values for rotation-minimizing frames defined by the equations (1) at $u=0$.

Finally we define the rotation-minimizing operator

$$
\mathcal{R}_{\mathbf{x}}: \quad F_{\mathbf{x}} \rightarrow \mathcal{C}\left(I, \mathbb{S}^{2}\right),
$$

where $\mathbb{S}^{2}$ is the unit sphere in $\mathbb{R}^{3}$, as the mapping which assigns to any initial vector $\mathbf{r}_{0} \in F_{\mathbf{x}}$ the solution of the equations (1) with initial value $\mathbf{r}(0)=\mathbf{r}_{0}$. Consequently, $\mathcal{R}_{\mathbf{x}}(u)$ is a mapping from $F_{\mathbf{x}}$ into $\mathbb{S}^{2}$ for any fixed value of the parameter $u \in I$.

\subsection{Möbius transformations}

We consider the three-dimensional space which is extended by adding a single point $\infty$ at infinity. For any sphere with radius $r>0$ and center $\mathbf{c}$, the mapping

$$
\rho: \mathbf{y} \mapsto \mathbf{c}+\frac{r^{2}}{\|\mathbf{y}-\mathbf{c}\|^{2}}(\mathbf{y}-\mathbf{c}), \quad \mathbf{y} \notin\{\mathbf{c}, \infty\},
$$

which additionally maps $\mathbf{c}$ to $\infty$ and vice versa, is said to be the inversion with respect to the sphere. It satisfies $\rho^{2}=\mathrm{id}$. The reflection at a plane is also considered as an inversion, which keeps $\infty$ fixed. A general Möbius transformation $\mu$ is defined as a composition of an arbitrary number of inversions with respect to spheres or planes. In particular, translations and rotations are special Möbius transformations, since they can be obtained by composing two reflections at planes.

The set of Möbius transformations forms a group with respect to composition, which possesses the Euclidean group as a subgroup. Möbius transformations are known to be the most general conformal (i.e., angle-preserving) transformations of the three-dimensional space. The image of any plane or sphere under a Möbius transformation is again a plane or a sphere.

While a Euclidean displacement provides six degrees of freedom, the set of Möbius transformations depends on 10 free parameters. These transformations can be represented by matrices of the group $O(4,1)$, which is a Lie group of dimension 10. Any Möbius transformation is also a birational mapping. See [1, $2,15]$ for additional information on this interesting class of transformations. 
The differential

$$
\mathrm{d} \mu_{\mathbf{y}}: \mathbb{R}^{3} \rightarrow \mathbb{R}^{3}
$$

of a Möbius transformation at a point $\mathbf{y}$ maps the tangent space of the three dimensional-space at any point $\mathbf{y}$ into the tangent space at $\mu(\mathbf{y})$. More precisely, for any vector $\mathbf{v}$ and any differentiable function $f: \mathbb{R}^{3} \rightarrow \mathbb{R}$, the directional derivative of $f$ with respect to the direction $\mathrm{d} \mu_{\mathbf{y}}(\mathbf{v})$ at the point $\mu(\mathbf{y})$ is equal to the directional derivative of $f \circ \mu$ with respect to the direction $\mathbf{v}$ at the point $\mathbf{y}$, see $[11]$.

In addition we define the normalized differential

$$
\mathrm{d}^{\star} \mu_{\mathbf{y}}: \mathbb{R}^{3} \rightarrow \mathbb{R}^{3}: \mathbf{v} \mapsto \frac{\|\mathbf{v}\|}{\left\|\mathrm{d} \mu_{\mathbf{y}}(\mathbf{v})\right\|} \mathrm{d} \mu_{\mathbf{y}}(\mathbf{v})
$$

as the version of this mapping which preserves the length of all vectors $\mathbf{v}$.

As a special case we consider the inversion $\phi$ with respect to the unit sphere,

$$
\phi: \mathbf{y} \mapsto \frac{1}{\|\mathbf{y}\|^{2}} \mathbf{y}, \quad \mathbf{y} \notin\{\mathbf{0}, \infty\}
$$

The differential $\mathrm{d} \phi_{\mathbf{y}}$ of this inversion is

$$
\mathrm{d} \phi_{\mathbf{y}}: \mathbb{R}^{3} \rightarrow \mathbb{R}^{3}: \mathbf{v} \mapsto \frac{\|\mathbf{y}\|^{2} \mathbf{v}-2(\mathbf{v} \cdot \mathbf{y}) \mathbf{y}}{\|\mathbf{y}\|^{4}}
$$

A short computation confirms that the length of the image of a vector equals

$$
\left\|\mathrm{d} \phi_{\mathbf{y}}(\mathbf{v})\right\|=\frac{\|\mathbf{v}\|}{\|\mathbf{y}\|^{2}}
$$

We use this result to find the normalized differential which preserves the length of the vectors,

$$
\mathrm{d}^{\star} \phi_{\mathbf{y}}(\mathbf{v}): \mathbb{R}^{3} \rightarrow \mathbb{R}^{3}: \mathbf{v} \mapsto\|\mathbf{y}\|^{2} \mathrm{~d} \phi_{\mathbf{y}}(\mathbf{v})=\mathbf{v}-\frac{2(\mathbf{v} \cdot \mathbf{y})}{\|\mathbf{y}\|^{2}} \mathbf{y} .
$$

In the case of a general Möbius transformation, which is obtained by composing several inversions, the differential and the normalized differential are both linear transformations whose coefficients are quadratic rational functions of the coordinates of the point $\mathbf{y}$.

\subsection{Invariance of rotation-minimizing frames}

Möbius transformations commute with the computation of rotation minimizing frames. More precisely, one has the following result.

Theorem 1. Consider a Möbius transformation $\mu$ which maps the curve $\mathbf{x}$ into another regular curve segment $\mu \circ \mathbf{x}$. Then 


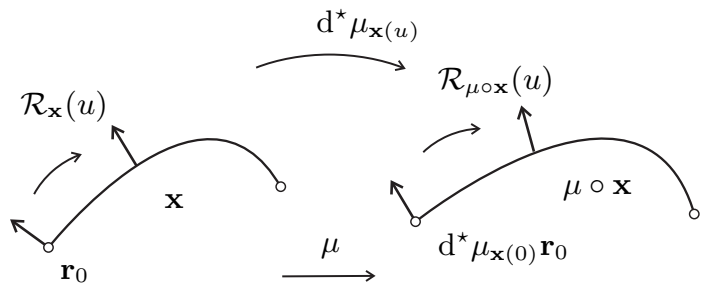

Fig. 1. Invariance of RMF under Möbius transformations, see Theorem 1.

(i) the evaluation of the rotation-minimizing vector field with respect to $\mathbf{x}$ at the parameter value $u$ for an initial vector $\mathbf{r}_{0}$ and the application of the normalized differential of $\mu$ to the resulting vector

gives the same result as

(ii) the evaluation of the rotation-minimizing vector field with respect to $\mu \circ \mathbf{x}$ at this parameter value $u$, where the initial vector is the image of $\mathbf{r}_{0}$ under the normalized differential of $\mu$ at $\mathbf{x}(0)$,

cf. Fig. 1. More precisely,

$$
\mathcal{R}_{\mu \circ \mathbf{x}}(u) \circ \mathrm{d}^{\star} \mu_{\mathbf{x}(0)}=\mathrm{d}^{\star} \mu_{\mathbf{x}(u)} \circ \mathcal{R}_{\mathbf{x}}(u) \quad \text { for all } u \in I,
$$

where $\mathcal{R}_{\mu \circ \mathbf{x}}$ and $\mathcal{R}_{\mathbf{x}}$ are the rotation-minimizing operators of the transformed and the original curve and $\mathrm{d}^{\star} \mu_{\mathbf{x}(u)}$ is the normalized differential of the Möbius transformation at $\mathbf{x}(u)$, respectively.

Proof. It suffices to prove this result for the inversion $\phi$ with respect to the unit sphere, since any Möbius transformation is a composition of inversions and the result is obviously true for uniform scalings and Euclidean displacements.

We consider a rotation-minimizing vector $\mathbf{v}$ of the given curve $\mathbf{x}$. Consequently, it satisfies the two conditions

$$
\mathbf{v} \cdot \mathbf{x}^{\prime}=0 \quad \text { and } \quad \mathbf{v}^{\prime}=\frac{\mathbf{v}^{\prime} \cdot \mathbf{x}^{\prime}}{\mathbf{x}^{\prime} \cdot \mathbf{x}^{\prime}} \mathbf{x}^{\prime}
$$

see (1). Using a direct computation one can show that

$$
\begin{array}{r}
\mathrm{d}^{\star} \phi_{\mathbf{x}(u)}(\mathbf{v}) \cdot(\phi \circ \mathbf{x})^{\prime}=\left(\mathbf{v}-\frac{2(\mathbf{v} \cdot \mathbf{x})}{\|\mathbf{x}\|^{2}} \mathbf{x}\right) \cdot\left(\frac{\|\mathbf{x}\|^{2} \mathbf{x}^{\prime}-2\left\langle\mathbf{x}^{\prime}, \mathbf{x}\right\rangle \mathbf{x}}{\|\mathbf{x}\|^{4}}\right)= \\
=\frac{1}{\|\mathbf{x}\|^{4}}\left(\|\mathbf{x}\|^{2}\left(\mathbf{v} \cdot \mathbf{x}^{\prime}\right)+(-2-2+4) \frac{\|\mathbf{x}\|^{2}\left(\mathbf{x}^{\prime} \cdot \mathbf{x}\right)(\mathbf{v} \cdot \mathbf{x})}{\|\mathbf{x}\|^{2}}\right)=0 .
\end{array}
$$

In addition we get that

$$
\begin{aligned}
& \left(\mathrm{d}^{\star} \phi_{\mathbf{x}(u)}(\mathbf{v})\right)^{\prime}=\left(\mathbf{v}-\frac{2(\mathbf{v} \cdot \mathbf{x})}{\|\mathbf{x}\|^{2}} \mathbf{x}\right)^{\prime}= \\
& \quad=\mathbf{v}^{\prime}-\left(\frac{2\left(\mathbf{v}^{\prime} \cdot \mathbf{x}\right)}{\|\mathbf{x}\|^{2}}+\frac{2\left(\mathbf{v} \cdot \mathbf{x}^{\prime}\right)}{\|\mathbf{x}\|^{2}}+\frac{4(\mathbf{v} \cdot \mathbf{x})\left(\mathbf{x} \cdot \mathbf{x}^{\prime}\right)}{\|\mathbf{x}\|^{4}}\right) \mathbf{x}-\frac{2(\mathbf{v} \cdot \mathbf{x})}{\|\mathbf{x}\|^{2}} \mathbf{x}^{\prime}
\end{aligned}
$$


After replacing $\mathbf{v}^{\prime}$ using (14) and rearranging one arrives at

$$
\left(\mathrm{d}^{\star} \phi_{\mathbf{x}(u)}(\mathbf{v})\right)^{\prime}=\|\mathbf{x}\|^{2}\left(\frac{\mathbf{v}^{\prime} \cdot \mathbf{x}^{\prime}}{\mathbf{x}^{\prime} \cdot \mathbf{x}^{\prime}}-\frac{2(\mathbf{v} \cdot \mathbf{x})}{\|\mathbf{x}\|^{2}}\right)(\phi \circ \mathbf{x})^{\prime} .
$$

Consequently, if $\mathbf{v}$ is a rotation-minimizing vector field with respect to the original curve $\mathbf{x}$, then the images $\mathrm{d}^{\star} \phi_{\mathbf{x}(u)}(\mathbf{v})$ form a rotation-minimizing vector field with respect to the image curve $\phi \circ \mathbf{x}$, since the conditions which are analogous to (14) are satisfied. This completes the proof, as the initial conditions at $u=0$ are linked by the normalized differential $\mathrm{d}^{\star} \phi_{\mathbf{x}(0)}$ at the first point of the curve.

Remark 1. Möbius transformations are also known to preserve the lines of curvature of surfaces. This observation can be used to derive a geometric proof of the RMF preservation, since the normal vectors of a surface along a curvature line form a rotation-minimizing vector field.

Remark 2. Another frame of a space curve, which is also preserved by Möbius transformations, can be obtained by assembling a triplet of vectors consisting of (1) the unit tangent vector, (2) the unit normal vector of the osculating sphere at the point of the curve, and (3) the cross product of these two vectors. This frame is generally not a rotation-minimizing frame. See also [3] for a discussion of conformally invariant frames of curves.

\subsection{Invariance of $\mathrm{PH}$ curves and rational RMFs}

In this section we consider the special case where $\mathbf{x}$ is a polynomial or rational curve. For any rational curve $\mathbf{x}$ and any rational vector field $\mathbf{r}(u)$, the curve $\mu \circ \mathbf{x}$ is again a rational curve and the vector field $u \mapsto \mathrm{d}^{\star} \mu_{\mathbf{x}(u)}(\mathbf{r}(u))$ is again a rational vector field. Indeed, Möbius transformations $\mu$ are birational mappings and their normalized differentials $\mathrm{d}^{\star} \mu_{\mathbf{y}}$ are linear transformations whose coefficients depend rationally on the point coordinates of $\mathbf{y}$.

Recall that a curve $\mathbf{x}$ is said to be a polynomial Pythagorean-hodograph $(\mathrm{PH})$ curve if the length $\left\|\mathbf{x}^{\prime}\right\|$ of its first derivative vector is a polynomial function of the parameter $u$ for $u \in I$. Following Ueda [20], we consider the extension of this notion to the rational case: a curve is said to be a rational PH curve if the length $\left\|\mathbf{x}^{\prime}\right\|$ of its first derivative vector is a rational function of the parameter $u$ for $u \in I$.

Corollary 1. If $\mathbf{x}$ is a polynomial PH curve, then $\mu \circ \mathbf{x}$ is a rational PH curve. Moreover, if $\mathbf{x}$ has a rotation-minimizing frame which is described by rational vector fields $(\mathbf{t}(u), \mathbf{r}(u), \mathbf{s}(u))$, then $\mu \circ \mathbf{x}$ possesses the rational rotationminimizing frame

$$
\left(\mathrm{d}^{\star} \mu_{\mathbf{x}(u)} \mathbf{t}(u), \mathrm{d}^{\star} \mu_{\mathbf{x}(u)} \mathbf{r}(u), \mathrm{d}^{\star} \mu_{\mathbf{x}(u)} \mathbf{s}(u)\right)
$$

which is obtained by applying the differential $\mathrm{d}^{\star} \mu_{\mathbf{x}(u)}$ to these vector fields. 
Proof. The first part of the corollary is a direct consequence of the properties of the normalized differential. The second one follows by combining this observation with Theorem 1.

The first part of this corollary was also noted by Ueda [20, §2]. The degree of a $\mathrm{PH}$ curves is doubled by applying a Möbius transformation.

\section{Interpolation by curves with rational RMF}

We describe an algorithm for Hermite interpolation by curves that posses a rational rotation-minimizing frame (i.e. an RMF which can be described by rational vector fields) demonstrate its performance by two examples.

\subsection{The algorithm}

Given a curve $\mathbf{x}$ with parameter $u$ and domain $I=[0,1]$, we generate a piecewise rational approximation of its rotation-minimizing frame with $k$ segments, which is globally $G^{1}$, as follows (see also Fig. 2).

1. The curve is split into $k$ segments, $u \in\left[u_{i-1}, u_{i}\right]$, where $u_{j}=j / k$ (Fig. 2a).

2. For each segment we generate the unique sphere which passes through $\mathbf{x}\left(u_{i-1}\right)$ and $\mathbf{x}\left(u_{i}\right)$ and which also touches the tangents of the curves at these points. Next we choose a Möbius transformation $\mu_{i}$ which transforms this sphere into a plane $\pi_{i}$ and apply it to the curve (Fig. 2b,c).

3 . For each segment we generate a $\mathrm{PH}$ cubic in the plane $\pi_{i}$ which interpolates the $G^{1}$ Hermite boundary data at $\left(\mu_{i} \circ \mathbf{x}\right)\left(u_{i-1}\right)$ and $\left(\mu_{i} \circ \mathbf{x}\right)\left(u_{i}\right)$ and compute its rational rotation-minimizing frame (Fig. 2d).

4. We apply the inverse Möbius transformations $\mu_{i}^{-1}$ to the planar PH cubics and to their rational rotation-minimizing frames (Fig. 2e,f).

The last three steps steps are now explained in more detail.

Step 2. There are two degrees of freedom for choosing the point $\mathbf{f}_{i}$ on the sphere which is mapped into $\infty$ by the Möbius transformation. We choose it as the point on the sphere which possesses the maximum distance to $\mathbf{x}\left(u_{i-1}\right)$ and $\mathbf{x}\left(u_{i}\right)$, in order to obtain a mapping which possesses a relatively small distortion in the region of interest. We define the image plane $\pi_{i}$ of the sphere as the unique plane which passes through $\mathbf{x}\left(u_{i-1}\right)$ and $\mathbf{x}\left(u_{i}\right)$ and has the normal vector $\mathbf{f}_{i}-\mathbf{z}_{i}$, where $\mathbf{z}_{i}$ is the center of the sphere.

Step 3. The Bézier form of PH cubics is characterized by a simple geometric condition on the shape of the control polygon [10]. Using this observation, an algorithm for $G^{1}$ Hermite interpolation can be formulated [13,17], which finds the interpolating $\mathrm{PH}$ cubic by solving a single quadratic equation. Note that this equation does not always possess real solutions. However, if the data are sampled from a smooth curve with sufficiently small step size (i.e., sufficiently 


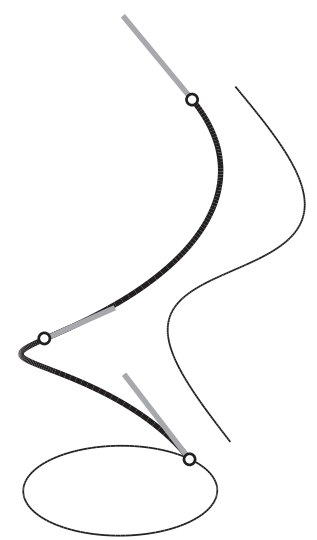

(a)

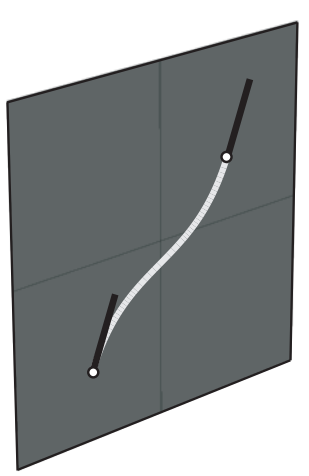

(d)

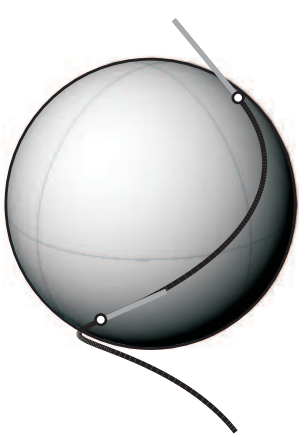

(h)

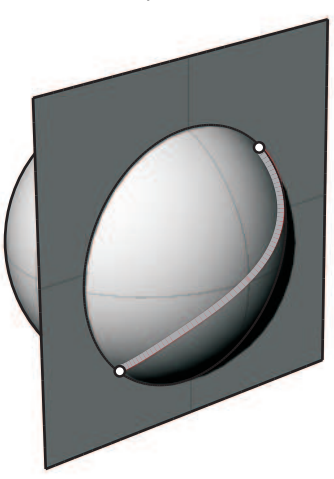

(e)

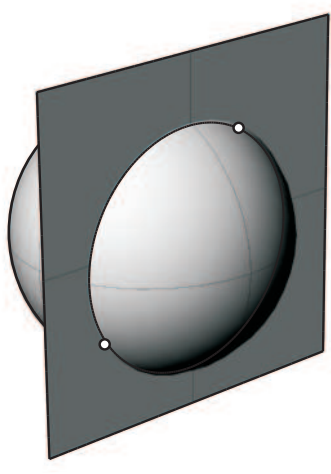

(c)

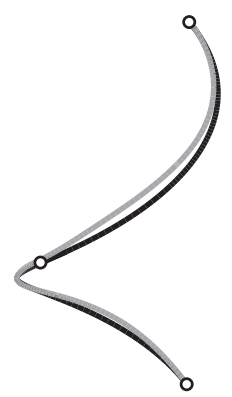

(f)

Fig. 2. The algorithm for computation of a rational approximation of the rotationminimizing frame, applied to a helix, $k=2$. (a) segmentation, (b,c) defining the Möbius transformation, (d) planar Hermite interpolation, (e) inverse Möbius transformation and (f) helix with approximating curves.

large $k$ ), then real solutions exist and to produce a unique Hermite interpolant with approximation order 4 . This can be shown by adapting the analysis in the proof of [16, Theorem 10].

Finally we generate the rational rotation-minimizing frame of a planar $\mathrm{PH}$ cubic $\mathbf{c}_{i}$,

$$
\left(\mathbf{c}_{i}^{\prime} /\left\|\mathbf{c}_{i}^{\prime}\right\|, \mathbf{n}, \mathbf{c}_{i}^{\prime} /\left\|\mathbf{c}^{\prime}\right\| \times \mathbf{n}\right),
$$

where $\mathbf{n}$ is a unit normal vector of the plane.

Step 4. By applying the inverse Möbius transformation $\mu_{i}^{-1}$ to $\mathbf{c}_{i}$ we obtain the spherical rational $\mathrm{PH}$ curve $\mu_{i}^{-1} \circ \mathbf{c}_{i}$ of degree 6 . Its rational rotation-minimizing frame consists of the unit tangent vector, the unit normal vector field of the sphere, and of the cross product of these two vectors. 

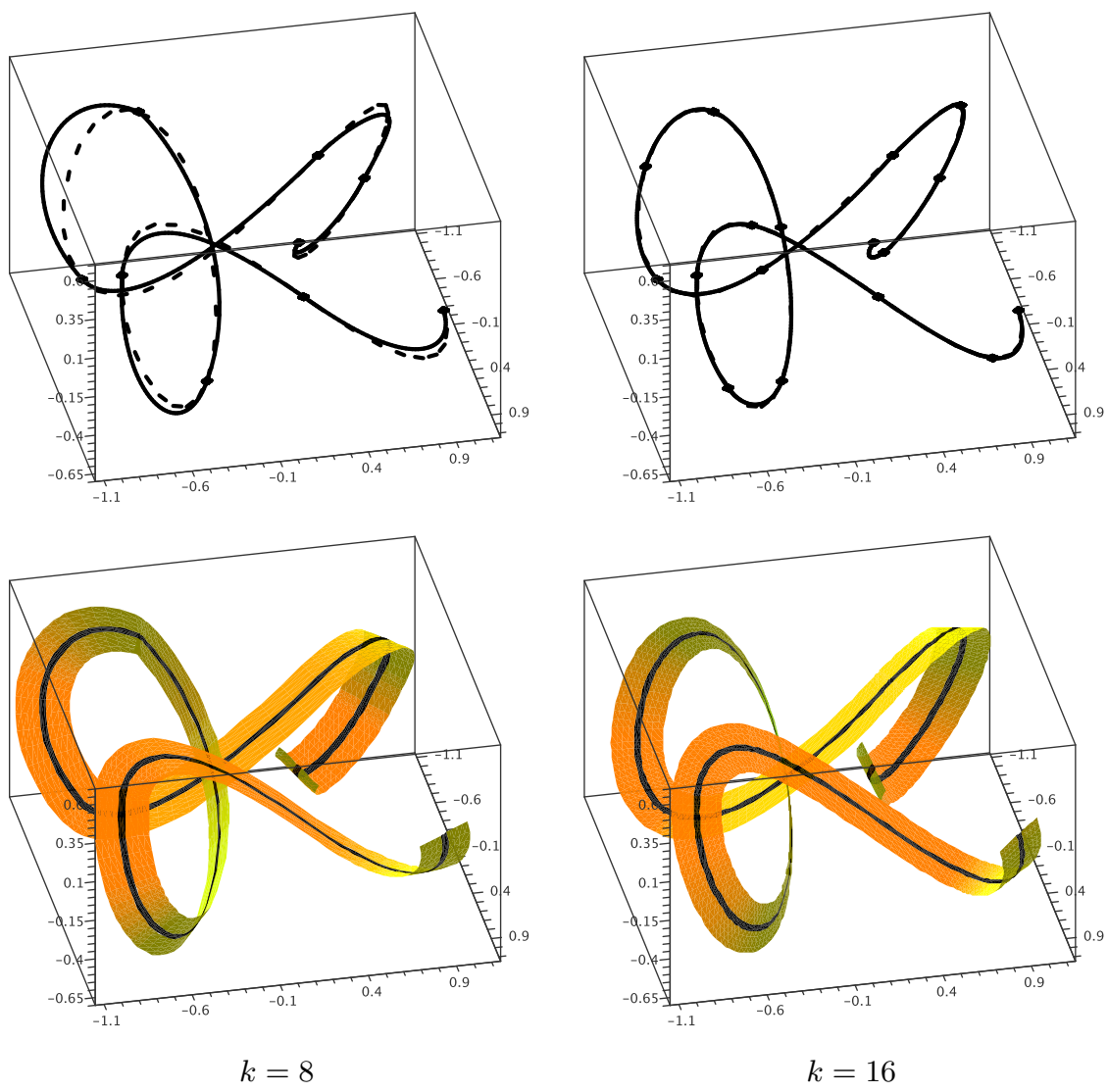

Fig. 3. Top row: Approximation of a space curve (dashed) by rational curves with rational rotation minimizing frames (solid), which were obtained by our algorithm with $k=8$ (left) and $k=16$ (right) segments. Bottom row: The strips of developable surfaces which are generated by a vector of the RMF for both approximations.

\section{$3.2 \quad$ Examples}

Example 1. We apply the algorithm to the curve segment

$$
\mathbf{x}(t)=\left(\sin (2 t) \cos (t)+\frac{1}{4} \sin (t), \sin (2 t) \sin (t)+\frac{1}{4} \cos (t), \frac{1}{2} \sin (4 t)\right),
$$

with domain $t \in\left[\frac{\pi}{4}, 2 \pi\right]$. The approximating rational curves with rational RMF and the developable surface strips generated by a vector of the piecewise rational RMF are shown in Figure 3. The approximation with $k=8$ segments has still a relatively large distance to the original curve (dashed). If an approximation with $k=16$ segments is used, then both curves can no longer be visually distinguished. 


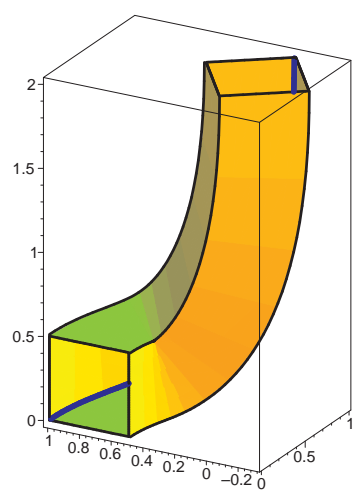

\begin{tabular}{|c|c|c|c|c|}
\hline$k$ & $A_{k}$ & $\frac{A_{k / 2}}{A_{k}}$ & $E_{k}$ & $\frac{E_{k / 2}}{E_{k}}$ \\
\hline 1 & $2.3710^{-1}$ & $n / a$ & $2.7610^{-1}$ & $n / a$ \\
\hline 2 & $3.1710^{-2}$ & 7.47 & $2.6710^{-2}$ & 10.3 \\
\hline 4 & $6.8610^{-3}$ & 4.62 & $2.0110^{-3}$ & 13.3 \\
\hline 8 & $6.2910^{-4}$ & 10.91 & $1.3210^{-4}$ & 15.2 \\
\hline 16 & $1.0110^{-4}$ & 6.21 & $8.3510^{-6}$ & 15.8 \\
\hline 32 & $1.3010^{-5}$ & 7.80 & $5.2410^{-7}$ & 15.9 \\
\hline
\end{tabular}

Fig. 4. Example 2: Sweep surface generated by the non-rational RMF of a space PH cubic (left). Average and maximum error for different numbers of segments (right).

Example 2. In order to compare the interpolant which is computed using the new method with the rotation-minimizing frame of tge original curve, we applied the algorithm to the space $\mathrm{PH}$ cubic

$$
\mathbf{x}(t)=\left(3 t(1-t)^{2}+3 t^{2}(1-t)+t^{3},(1-t)^{3}+3 t(1-t)^{2}, 2 t^{3}\right)
$$

with domain $t \in[0,1]$. As observed in [7], $\mathrm{PH}$ cubics are equipped with rational Frenet frames and the difference angle to the (generally non-rational) rotationminimizing frame can be computed from

$$
\theta(t)=\theta_{0}-\int_{0}^{t} \tau(u)\left\|\mathbf{x}^{\prime}(u)\right\| \mathrm{d} u,
$$

since both the torsion $\tau(u)$ of $\mathbf{x}$ and the parametric speed $\left\|\mathbf{x}^{\prime}(u)\right\|$ are rational functions of $u$. Fig. 4 shows the rotation-minimizing frame of (21).

In order to analyze the error, we consider the length of the differences

$$
\delta(u)=\left\|\mathbf{r}_{\text {approx }}(u)-\mathbf{r}\left(u^{*}(u)\right)\right\|,
$$

where each point of the approximating curve is compared with the nearest point of the original curve,

$$
u^{*}(u)=\arg \min _{v \in[0,1]}\left\|\mathbf{x}_{\text {approx }}(u)-\mathbf{x}(v)\right\| .
$$

We use numerical integration to evaluate the average error and the end point error,

$$
A_{k}=\int_{0}^{1} \delta(u) \mathrm{d} u \quad \text { and } \quad E_{k}=\delta(1)
$$

for different numbers of segments $k$. The results are reported in the table in Fig. 4. The numbers indicate that the average error decreases as $h^{3}$, where $h=2^{-k}$ is the stepsize, while the error at the joints - and in particular at the end point of the curve - decreases faster with order 4 (in accordance with the phenomenon which is described in $[22,23])$. 


\section{Conclusion}

We showed that rotation-minimizing frames of space curves are invariant under Möbius transformations. Since these transformations also preserve rational frames and rational $\mathrm{PH}$ curves, it was possible to use this result in order to formulate an algorithm for $G^{1}$ Hermite interpolation by rational curves with rational rotation-minimizing frames. As a possible topic for future research, one might try to use the recent results about $\mathrm{PH}$ quintics with rational rotationminimizing frames [9] to achieve higher geometry flexibility, which might lead to a scheme for interpolation with a higher order of smoothness.

Acknowledgment The authors would like to thank Martin Peternell for useful discussions. The first author was support by grant no. P17387-N12 of the Austrian Science Fund (FWF).

\section{References}

1. L. V. Ahlfors, Möbius transformations in $\mathbb{R}^{n}$ expressed through $2 \times 2$ matrices of complex numbers, Complex Variables 2 (1986), 215-224.

2. A. F. Beardon, Continued Fractions, Möbius transformations and Clifford Algebras, Bull. London Math. Society 35 (2003), 302-308.

3. G. M. Beffa, Poisson brackets associated to the conformal geometry of curves, Trans. Amer. Math. Society 357 (2005), 2799-2827.

4. R. Bishop, There is more than one way to frame a curve, Amer. Math. Monthly, 82.3 (1975), 246-251.

5. H. I. Choi and C. Y. Han, Euler-Rodrigues frames on spatial Pythagorean-hodograph curves, Comput. Aided Geom. Design 19 (2002), 603-620.

6. H. I. Choi, D. S. Lee, H. P. Moon, Clifford algebra, spin representation and rational parametrization of curves and surfaces, Adv. Comput. Math. 17 (2002), 5-48.

7. R. T. Farouki, Exact rotation-minimizing frames for spatial Pythagorean-hodograph curves, Graphical Models 64 (2002), 382-395.

8. R. T. Farouki, Pythagorean-Hodograph Curves: Algebra and Geometry Inseparable, Springer, Berlin 2008.

9. R. T. Farouki, C. Giannelli, C. Manni and A. Sestini, Quintic space curves with rational rotation-minimizing frames, manuscript, 2008, available at http:// mae.ucdavis.edu/ farouki/.

10. R. T. Farouki and T. Sakkalis, Pythagorean-hodograph space curves, Adv. Comput. Math. 2 (1994), 41-66.

11. T. Frankel, The Geometry of Physics, Cambridge University Press, 1999.

12. C. Y. Han, Nonexistence of rational rotation-minimizing frames on cubic curves, Comput. Aided Geom. Design 6 (2008), 77-78.

13. B. Jüttler and C. Mäurer, Cubic Pythagorean Hodograph Spline Curves and Applications to Sweep Surface Modeling, Comput. Aided Design 31 (1999), 73-83.

14. B. Jüttler, Generating rational frames of space curves via Hermite interpolation with Pythagorean hodograph cubic splines, in Geometric Modelling and Processing '98, Bookplus Press, Soul 1998, 83-106.

15. S. Leopoldseder, Algorithms on cone spline surfaces and spatial osculating arc splines, Comput. Aided Geom. Design 18 (2001), 505-530. 
16. C. Mäurer and B. Jüttler. Rational approximation of rotation minimizing frames using Pythagorean-hodograph cubics. J. Geom. Graph., 3(2):141-159, 1999.

17. D. S. Meek, D. J. Walton, Geometric Hermite interpolation with Tschirnhausen cubics, J. Comput. Appl. Math. 81 (1997), 299-309.

18. H. Pottmann and M. Wagner, Principal Surfaces. In: T.N.T. Goodman and R.R. Martin (eds.), The Mathematics of Surfaces VII, Information Geometers, Winchester, 337-362, 1997.

19. H. Pottmann and M. Wagner Contributions to Motion Based Surface Design. Int. J. of Shape Modeling 4, 183-196, 1998.

20. K. Ueda, Spherical Pythagorean-Hodograph Curves, In: M. Dæhlen, T. Lyche, and L. L. Schumaker (eds.), Mathematical Methods for Curves and Surfaces II, Vanderbilt University Press, Nashville 1998, 485 - 492.

21. M. Wagner and B. Ravani, Curves with Rational Frenet-Serret motion, Comput. Aided Geom. Design 15 (1997), 79-101.

22. W. Wang and B. Joe, Robust computation of the rotation minimizing frame for sweep surface modeling, Comput. Aided Design 29 (1997), 379-391.

23. W. Wang, B. Jüttler, D. Zheng and Y. Liu, Computation of Rotation Minimizing Frame, ACM Trans. on Graphics 27.1 (2008), article no. 2. 